\title{
Comparison of Cognitive Performance and Cardiac Function Between Three Different Rat Models of Vascular Dementia
}

\author{
Baoyu Wei ${ }^{1}$ \\ Shihao Wu' \\ Zhaoqi Wang' \\ Wanshan Song ${ }^{2}$ \\ Jinqiang Zhu'
}

'State Key Laboratory of ComponentBased Chinese Medicine, Institute of Traditional Chinese Medicine, Tianjin University of Traditional Chinese Medicine, Tianjin, 301617, People's Republic of China; ${ }^{2}$ Department of Acupuncture and Cerebropathy, Second Affiliated Hospital of Tianjin University of Traditional Chinese Medicine, Tianjin, 300250, People's Republic of China
Correspondence: Wanshan Song; Jinqiang

Zhu

Email sws19870604@I63.com;

zhujinqiangl860@।63.com
Purpose: Establishing an ideal animal model is essential for studying the pathogenesis, prevention and treatment of vascular dementia (VD). The present study was designed to compare the differences of behavior, cerebral blood flow (CBF), cardiac output and the levels of myocardial enzyme of three different VD rat models.

Methods: The rats were randomly divided into sham-operated group (SHAM), permanent bilateral common carotid artery occlusion group (BCCAO), BCCAO combined with sodium nitroprusside $\left(2.0 \mathrm{mg} \cdot \mathrm{kg}^{-1}\right)$ group $(\mathrm{BCCAO}+2.0 \mathrm{SNP})$ and $\mathrm{BCCAO}$ combined with sodium nitroprusside $\left(2.5 \mathrm{mg} \cdot \mathrm{kg}^{-1}\right)$ group $(\mathrm{BCCAO}+2.5 \mathrm{SNP})$. After operation, Morris water maze test, echocardiographic evaluation and the measurement of $\mathrm{CBF}$ were performed, then the levels of myocardial enzymes in serum were assessed during euthanasia.

Results: Compared with SHAM rats, the three VD model rats showed different degrees of cognitive impairment, lower cardiac output and $\mathrm{CBF}$, and BCCAO rats showed higher levels of myocardial enzymes. Compared with BCCAO rats, the spatial learning ability of BCCAO+2.0SNP rats and $\mathrm{BCCAO}+2.5 \mathrm{SNP}$ rats was more severely impaired, while the levels of myocardial enzymes of $\mathrm{BCCAO}+2.0 \mathrm{SNP}$ rats were lower. Compared with $\mathrm{BCCAO}+2.0 \mathrm{SNP}$ rats, $\mathrm{BCCAO}$ $+2.5 \mathrm{SNP}$ rats showed no significant difference in cognitive function and cardiac function.

Conclusion: Our present study demonstrated that all of the three different VD rat models exhibited cognitive and cardiac function impairment. The BCCAO+2.0SNP model and BCCAO $+2.5 \mathrm{SNP}$ model damaged the spatial learning ability more seriously. The BCCAO+2.5SNP model caused more comprehensive cognitive impairment. In addition, the BCCAO+2.0SNP model and BCCAO+2.5SNP model might cause more serious damage to cardiac function.

Keywords: vascular dementia rat models, permanent bilateral common carotid artery occlusion, sodium nitroprusside, cognitive performance, cardiac function

\section{Introduction}

Vascular dementia (VD), a complex neurological disease that affects memory and cognitive abilities, is a major contributor to the dementia after Alzheimer's disease, accounting for at least $20 \%$ of dementia cases worldwide. ${ }^{1} \mathrm{VD}$ is generally determined to be caused by impaired blood flow to the brain and damage to the blood vessels resulting from events such as stroke, ${ }^{2}$ and most of its current animal models are based on cerebral ischemia. ${ }^{3}$ However, so far, no animal model can comprehensively mimic the pathogenesis of VD. Therefore, choosing the appropriate model according to the purpose of the research is challenging but of crucial importance. 
The rat permanent bilateral common carotid artery occlusion (BCCAO) is the most-used method for modeling VD, which leads to chronic hypoperfusion and white matter injury. ${ }^{4}$ However, due to the abundant collateral circulation in rats, it is difficult to significantly reduce cerebral blood flow (CBF) only by ligating the bilateral common carotid arteries, so that the state of global cerebral ischemia cannot be fully achieved. ${ }^{5}$ Sodium nitroprusside (SNP), a nitric oxide donor, induces hypotension by dilating blood vessels, resulting in a lower $\mathrm{CBF}^{6,7}$ Accordingly, the BCCAO model was modified via combining with intraperitoneal administration of SNP. ${ }^{8}$ However, the dosage of SNP in experiments is inconsistent, and the frequently used dosage is $2.0 \mathrm{mg} \cdot \mathrm{kg}^{-1}$ and $2.5 \mathrm{mg} \cdot \mathrm{kg}^{-1} .9,10$ Although studies have focused on the mortality of rats at these two doses, there are few reports on the difference in cognitive performance.

Furthermore, based on the increasing studies on the cardiac-brain axis, more and more attention has been paid to the bi-directional feedback interactions between the heart and the brain. ${ }^{11}$ Not only the impaired cardiac function affects cerebral structure and functional capacity but also neuronal signals affect the cardiovascular continuum. ${ }^{12}$ However, there is no study to investigate the changes of cardiac function in VD rat model. Therefore, we designed this experiment to compare the differences in behavior, $\mathrm{CBF}$, cardiac output and the content of myocardial enzyme of three different VD rat models that including the BCCAO model, BCCAO combined with SNP $\left(2.0 \mathrm{mg} \cdot \mathrm{kg}^{-1}\right)$ model and BCCAO combined with SNP $\left(2.5 \mathrm{mg} \cdot \mathrm{kg}^{-1}\right)$ model, in order to evaluate their characteristics of cognitive impairment and impaired cardiac function after cerebral ischemia.

\section{Materials and Methods}

\section{Animals}

Male Sprague-Dawley rats (aged six-weeks, weighing 200 $\pm 10 \mathrm{~g}$ ) were purchased from Huafukang Bioscience Co. INC. (Certificate No. SCXK Beijing: 2019-0008) and acclimatized to the laboratory environment for 1 week. The rats were housed in cages under controlled temperature $\left(23 \pm 1^{\circ} \mathrm{C}\right)$ and humidity $(55 \pm 5 \%)$ with a $12 \mathrm{~h}$ light/dark cycle. Food and water were freely available throughout the study. All animal procedures were performed in strict accordance with the recommendations of the Provisions and General Recommendations of Chinese Experimental Animal.

Forty-four rats were divided at random into the following four groups: sham-operated group (SHAM), BCCAO group (BCCAO), BCCAO combined with SNP $\left(2.0 \mathrm{mg} \cdot \mathrm{kg}^{-1}\right)$ group $(\mathrm{BCCAO}+2.0 \mathrm{SNP})$ and $\mathrm{BCCAO}$ combined with SNP $\left(2.5 \mathrm{mg} \cdot \mathrm{kg}^{-1}\right)$ group (BCCAO+2.5SNP). The allocation of animals in each group is shown in Table 1.

\section{Surgery}

The surgical procedure was performed as described by previously. ${ }^{4,13,14}$ In brief, all rats had free access to water but not food for the $12 \mathrm{~h}$ before the surgery. The rats were anesthetized with a mixture of $5 \%$ isoflurane and $95 \%$ oxygen, and then a respiratory mask was used to supply a mixture of $3 \%$ isoflurane and $97 \%$ oxygen during the surgery. Through a midline cervical incision, both common carotid arteries were carefully exposed and gently separated from the cervical sympathetic and vagal nerves. In the BCCAO group, each common carotid artery was ligated with a 3-0 typed surgical silk suture, and then cauterized from the middle of the two silk sutures. The rats subjected to BCCAO combined with SNP (2.0mg $\cdot \mathrm{kg}^{-1}$ or $2.5 \mathrm{mg} \cdot \mathrm{kg}^{-1}$; Lot Number: 19110512 , China Resources Double-Crane Pharmaceutical CO., LTD., Beijing, China) had the same procedures, except for intraperitoneal administration of SNP before the common carotid artery was isolated and ligated. As sham-operated control group, the animals received the same operation without carotid artery ligations and without injection of SNP. During the surgical procedure, the body temperature of each rat was maintained at $37^{\circ} \mathrm{C}$ with a heating pad. All efforts were made to minimize animal suffering. After surgery, all rats were allowed to recover for 7 days.

\section{Morris Water Maze Test}

The spatial learning ability and reference memory of all rats were evaluated by Morris water maze (MWM) test as described previously at 7 days after the surgery. ${ }^{15}$ In a circular pool $(180 \mathrm{~cm}$ in diameter and $50 \mathrm{~cm}$ high) filled with $30 \mathrm{~cm}$ water at $23 \pm 2{ }^{\circ} \mathrm{C}$, the pool was divided into four equal zones (northeast, northwest, southeast and southwest). For the record of all trials, a video camera was mounted above the center of the tank.

Table I Information of Animal Numbers

\begin{tabular}{|l|c|c|c|c|}
\hline $\begin{array}{l}\text { Animal } \\
\text { Groups }\end{array}$ & Beginning & $\begin{array}{c}\text { Died During } \\
\text { Experiment }\end{array}$ & End & Experiment \\
\hline SHAM & 11 & 3 & 8 & CBF \\
BCCAO & 11 & 3 & 8 & Surgery \\
BCCAO+2.0SNP & 11 & 2 & & Surgery \\
& & 1 & & Behavior \\
BCCAO+2.5SNP & 11 & 3 & 8 & CBF \\
& & 3 & 7 & Surgery \\
\hline
\end{tabular}


During the acquisition training period of the task, the circular plastic platform $(15 \mathrm{~cm}$ in diameter) was placed in the middle of the northwest quadrant and submerged $2.0 \mathrm{~cm}$ below the water surface throughout training. The rats were gently released into the water by facing the tank wall at four different starting positions equally spaced around the perimeter of the pool. The rats were given a maximum of $60 \mathrm{~s}$ to find the hidden platform. Once the rat had found the platform, it was left for 10s to orient and then was removed, dried with a towel. If the platform was not found after 60 s, the rat was gently guided to it for $10 \mathrm{~s}$, then the training was terminated, and a maximum score of $60 \mathrm{~s}$ was assigned. The rats were subjected to four trials per day for 4 consecutive days. Escape latency, swimming distance and swimming speed before going on platform were collected.

To assess the spatial memory, a probe trial was performed $24 \mathrm{~h}$ after the last training trial. In the probe trial, the animals were allowed to swim freely in the pool removed from the platform for twice (once for $60 \mathrm{~s}$ ), with an interval of 30s. The number of times across the platform, time stayed in target quadrant and distance moved in target quadrant were recorded.

\section{Echocardiographic Study}

Echocardiographic evaluation of the left ventricular (LV) function was performed using a color Doppler ultrasound system (Vevo 3100; FUJIFILM VisualSonics, Inc., Canada) equipped with MS-250, 16.0-21.0 MHZ multifrequency transducer after MWM test. Rats were continuously anesthetized with $2 \%$ isoflurane combined with $98 \%$ oxygen, and then an optimal parasternal long-axis view (ie visualization of both the mitral and aortic valves and maximum distance between the aortic valve and the cardiac apex) was achieved. ${ }^{16}$ Guided by B-model, M-mode tracings were obtained by placing the sampling line at the level of the chordae of the mitral valve, perpendicular to the ventricular septum and the posterior wall of the left ventricle. All LV structures were manually measured by the same observer who was trained in advance. The measurements obtained were the mean of at least five cardiac cycles on the M-mode tracings. The following structural variables were measured: LV diastolic and systolic septal wall thickness (DSWT and SSWT, respectively), LV diastolic and systolic diameter (LVDD and LVSD, respectively), LV diastolic and systolic posterior wall thickness (DPWT and SPWT, respectively). LV function was assessed by the following parameters: LV ejection fraction (EF), LV endocardial fractional shortening (FS), LV mass, LV end-diastolic volume (EDV) and LV end-systolic volume (ESV).

\section{Measurement of Cerebral Blood Flow}

After the echocardiographic evaluation, $\mathrm{CBF}$ of all rats was measured with a non-invasive and non-contact laser Doppler imager (LDI2; Moor Instruments, Axminster, UK) ${ }^{17}$ Each rat was anesthetized with intraperitoneal injection of chloral hydrate $\left(300 \mathrm{mg} \cdot \mathrm{kg}^{-1}\right)$. Rectal temperature was maintained at approximately $37.0^{\circ} \mathrm{C}$ with a heating pad. The scalp was exposed after removing the head fur non-invasively with cream hair remover. The rat was moved below the scanning port of the imager, and the scanning distance was set to $53 \mathrm{~cm}$. CBF was imaged with a scan range of $4.2 \mathrm{~cm} \times 4.2 \mathrm{~cm}$ and a scan speed of $4 \mathrm{~ms} \cdot \mathrm{pixel}^{-1}$. All CBF images were sampled and measured by the same staff using a proportional sampling frame.

\section{The Levels of Myocardial Enzymes in Serum}

The blood was collected through the abdominal aorta of each rat and centrifuged at $3000 \mathrm{r} \cdot \mathrm{min}^{-1}$ for $10 \mathrm{~min}$ to separate the serum. An automatic biochemical analyzer (7020 ISE; Hitachi, Ltd., Japan) was used to measure the levels of myocardial enzymes and four blood lipid items in serum. The measured indicators were as follows: creatine kinase (CK), creatine kinase-MB (CK-MB), lactate dehydrogenase (LDH) and aspartate aminotransferase (AST).

\section{Statistical Analysis}

Values presented in the study were expressed as mean \pm SEM. Escape latency, swimming distance and swimming speed in MWM test were input to SPSS software (version 25.0) and analyzed by repeated measures two-way ANOVA with LSD post hoc test. The remaining non-repeated data were input into Excel 2016 and analyzed using a two-tailed Student's test. In all statistical comparison, $\mathrm{p}<0.05$ was used as significant. All graphs in this manuscript were constructed using Graph Pad Prism (version 6.0).

\section{Results}

\section{Morris Water Maze Test}

In MWM test, with the prolongation of training time, the escape latency, swimming distance and swimming speed became shortened/decrease in each group, except for the BCCAO+2.5SNP group, which was significantly longer/ increase on the fourth day (Figure 1A-C). Compared with SHAM group, the escape latency of the three VD model groups was significantly prolonged ( $\mathrm{p}<0.05$ or $\mathrm{p}<0.001$ ). The swimming distance of $\mathrm{BCCAO}+2.0 \mathrm{SNP}$ group and 

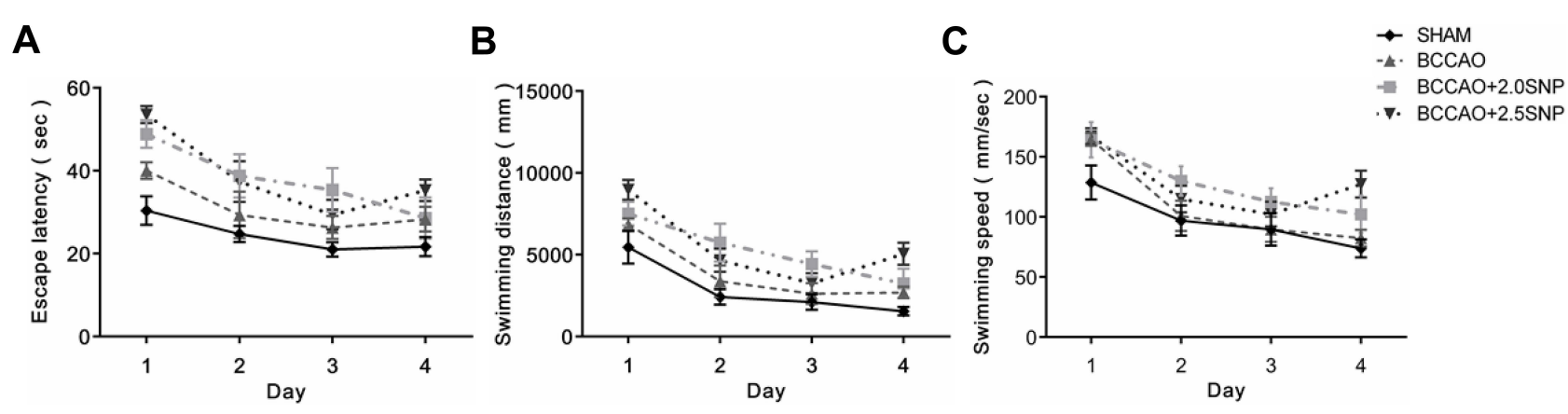

D

E
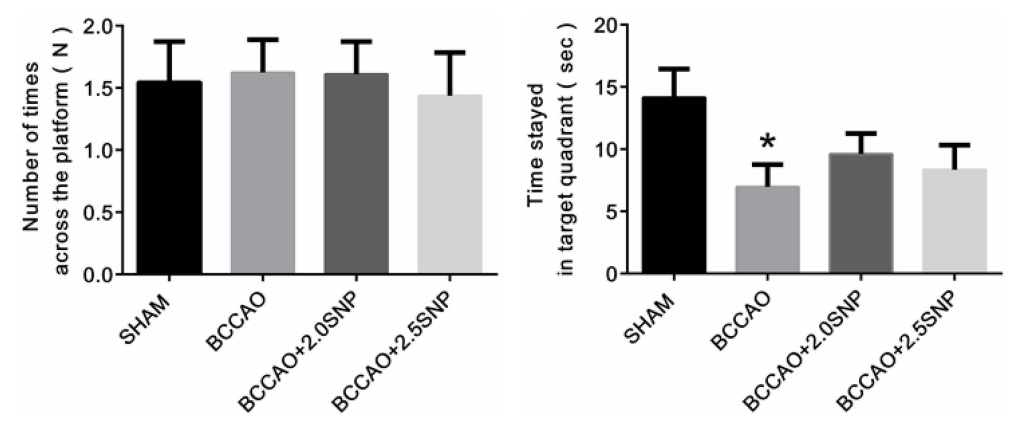

$\mathbf{F}$

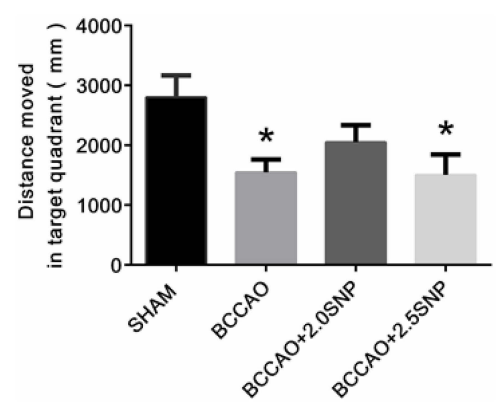

Figure I The spatial learning and reference memory of rats were investigated in Morris water maze. (A) Escape latency. (B) Swimming distance. (C) Swimming speed. (D) Number of times across the platform. (E) Time stayed in target quadrant. (F) Distance moved in target quadrant. The values are presented as means \pm SEM. vs SHAM, $* P<0.05$. SHAM: $n=1$; BCCAO: $n=8 ; B C C A O+2.0 S N P: n=9 ; B C C A O+2.5 S N P: n=8$.

BCCAO+2.5SNP group was significantly prolonged $(\mathrm{p}<0.001)$, and the BCCAO group had a prolonged trend, but it was not statistically significant $(p>0.05)$. The swimming speed of BCCAO + 2.5SNP group showed a significant increase $(\mathrm{p}<0.05)$, while there was no significant difference in the other groups $(\mathrm{p}>0.05)$. Compared with BCCAO group, the $\mathrm{BCCAO}+2.0 \mathrm{SNP}$ group and $\mathrm{BCCAO}+2.5 \mathrm{SNP}$ group showed a significant prolongation in the escape latency and swimming distance $(\mathrm{p}<0.05)$, but there was no significant difference in the swimming speed $(\mathrm{p}>0.05)$. Compared with $\mathrm{BCCAO}+2.0 \mathrm{SNP}$ group, the escape latency, swimming distance and swimming speed of $\mathrm{BCCAO}+2.5 \mathrm{SNP}$ group were no significant difference $(\mathrm{p}>0.05)$.

In the probe test, the number of times across platforms was not significantly different in any group of animals ( $p>0.05$; Figure 1D). Compared with SHAM group, the time stayed in target quadrant of BCCAO group was significantly shorter $(\mathrm{p}<0.05)$, and the rats in the other groups had a shortening trend, but it was not statistically significant ( $\mathrm{p}>0.05$; Figure 1E). The distance moved in target quadrant of BCCAO group and $\mathrm{BCCAO}+2.5 \mathrm{SNP}$ group was significantly shorter $(\mathrm{p}<0.05)$, and the rats in $\mathrm{BCCAO}+2.0 \mathrm{SNP}$ groups had a shortening trend, but it was not statistically significant $(\mathrm{p}>0.05$; Figure $1 \mathrm{~F})$. Compared with BCCAO group, there was no significant difference in the time stayed in target quadrant and distance moved in target quadrant in the other groups ( $p>0.05$ ). Compared with $\mathrm{BCCAO}+2.0 \mathrm{SNP}$ group, the time stayed in target quadrant and distance moved in target quadrant of $\mathrm{BCCAO}+2.5 \mathrm{SNP}$ group were no significant difference $(p>0.05)$.

\section{Echocardiographic Study}

The measured structural variables are shown in Table 2. Representative images of echocardiographic study are shown in Figure 2A. Compared with SHAM group, the BCCAO group, BCCAO+2.0SNP group and BCCAO $+2.5 \mathrm{SNP}$ group showed lower EF and FS $(\mathrm{p}<0.05$, $\mathrm{p}<$ 0.01 , or $\mathrm{p}<0.001$; Figure $2 \mathrm{~B}$ and $\mathrm{C}$ ). The $\mathrm{BCCAO}+2.0 \mathrm{SNP}$ group and $\mathrm{BCCAO}+2.5 \mathrm{SNP}$ group showed larger EDV and ESV $(p<0.05$ or $p<0.01$; Figure $2 D$ and $E$ ), and the rats in BCCAO group tended to increase, but it was not statistically significant $(\mathrm{p}>0.05)$. Compared with BCCAO group, the EF, FS, EDV and ESV of rats in the other groups showed no significant difference $(\mathrm{p}>0.05)$. Compared with BCCAO $+2.0 \mathrm{SNP}$ group, the EF, FS, EDV and ESV of rats in $\mathrm{BCCAO}+2.5 \mathrm{SNP}$ group showed no significant difference $(p>0.05)$. There was no significant difference in LV mass of rats in each group $(\mathrm{p}>0.05$, Figure $2 \mathrm{~F})$. 
Table 2 Echocardiographic Data

\begin{tabular}{|l|c|c|c|c|}
\hline & SHAM $(\mathbf{n}=11)$ & BCCAO $(\mathbf{n}=\mathbf{8})$ & BCCAO+2.0SNP $(\mathbf{n}=8)$ & BCCAO+2.5SNP $(\mathbf{n}=\mathbf{8})$ \\
\hline DSWT $(\mathrm{mm})$ & $2.07 \pm 0.08$ & $1.93 \pm 0.08$ & $1.97 \pm 0.10$ & $1.64 \pm 0.07 * * * 1+1 /$ \\
SSWT $(\mathrm{mm})$ & $3.37 \pm 0.10$ & $3.09 \pm 0.19$ & $3.16 \pm 0.13$ & $2.75 \pm 0.13 * * * \$$ \\
LVDD $(\mathrm{mm})$ & $6.48 \pm 0.27$ & $6.72 \pm 0.26$ & $7.03 \pm 0.17$ & $7.13 \pm 0.22$ \\
LVSD (mm) & $3.10 \pm 0.28$ & $3.96 \pm 0.19 *$ & $4.29 \pm 0.17 * *$ & $4.49 \pm 0.18^{* * * *}$ \\
DPWT (mm) & $2.29 \pm 0.21$ & $2.00 \pm 0.27$ & $1.94 \pm 0.19$ & $1.95 \pm 0.06$ \\
SPWT $(\mathrm{mm})$ & $3.20 \pm 0.17$ & $3.11 \pm 0.12$ & $3.01 \pm 0.07$ & $3.05 \pm 0.08$ \\
\hline
\end{tabular}

Notes: The values are expressed as means \pm SEM. vs SHAM, ${ }^{*} \mathrm{P}<0.05, * * \mathrm{P}<0.0 \mathrm{I},{ }^{*} * * \mathrm{P}<0.00 \mathrm{I} ; \mathrm{vs} \mathrm{BCCAO},{ }^{*} \mathrm{P}<0.05 ; \mathrm{vs} \mathrm{BCCAO}+2.0 \mathrm{SNP},{ }^{\$} \mathrm{P}<0.05$.

Abbreviations: LV, left ventricle; DSWT, LV diastolic septal wall thickness; SSWT, LV systole septal wall thickness; LVDD, LV diastolic diameter; LVSD, LV systolic diameter; DPWT, LV diastolic posterior wall thickness; SPWT, LV systolic posterior wall thickness.

\section{Measurement of Cerebral Blood Flow}

Representative CBF images measured by using laser speckle are shown in Figure 3A. Compared with SHAM rats, the rats from the other groups showed a significant decrease in $\mathrm{CBF}$ $(\mathrm{p}<0.05$; Figure $3 \mathrm{~B})$. There was no significant difference in $\mathrm{CBF}$ between the $\mathrm{BCCAO}$ rats, $\mathrm{BCCAO}+2.0 \mathrm{SNP}$ rats and $\mathrm{BCCAO}+2.5 \mathrm{SNP}$ rats $(\mathrm{p}>0.05)$.

\section{The Levels of Myocardial Enzymes and Blood Lipids in Serum}

Compared with SHAM rats, the levels of CK, CK-MB, LDH and AST were higher in BCCAO rats $(\mathrm{p}<0.05$ or $\mathrm{p}<0.01$; Figure $4 \mathrm{~A}-\mathrm{D}$ ). There had an increasing tendency in BCCAO+2.5SNP rats, but it was not statistically significant $(\mathrm{p}>0.05)$; and had no significant difference in BCCAO+2.0SNP $(p>0.05)$. Compared with BCCAO rats, the levels of CK, CK-MB, LDH and AST were lower in $\mathrm{BCCAO+2.0SNP}$ rats $(\mathrm{p}<0.05, \mathrm{p}<0.01$, or $\mathrm{p}<0.001)$, while there was not different in $\mathrm{BCCAO}+2.5 \mathrm{SNP}$ rats. There was no significant difference in the levels of $\mathrm{CK}$, CK-MB, LDH and AST between BCCAO+2.0SNP group and $\mathrm{BCCAO}+2.5 \mathrm{SNP}$ group $(\mathrm{p}>0.05)$.

\section{Discussion}

In VD, chronic cerebral hypoperfusion induced by persistent and moderate decrease of $\mathrm{CBF}$ is regarded as the main drive of causing cognitive decline, which induces a progressive, dynamic process involving ischemia, hypoxia, oxidative stress and neuroinflammation. ${ }^{18,19}$ However, most of the currently established models of VD produced acute single or multiple infarctions, which may be suitable for studying transient ischemic, rather than chronic survival studies. ${ }^{20}$ The BCCAO model is well established. It can produce a chronic, global hypoperfusion state, and show impaired cognitive memory in various behavioral tests (eg MWM, 8-arm radial maze, T-maze and novel object recognition test) without major motor dysfunction. ${ }^{21}$ In the present study, the escape latency of BCCAO rats was prolonged, and the time stayed in target quadrant and distance moved in target quadrant were shortened, suggesting the spatial learning ability and reference memory were impaired at 7 days after surgery, which is consistent with previous studies. ${ }^{22,23}$ The occurrence of BCCAO resulted in a decrease in CBF, approximately $66 \%$ in the cortex and $48 \%$ in the hippocampus after $2.5 \mathrm{~h} .{ }^{24} \mathrm{CBF}$ can be restored $35 \%$ in the cortex and $26 \%$ in the hippocampus after one week, and the recovery of both structures can reach $81 \%$ by 3 months. ${ }^{25}$ Our study showed that the CBF of BCCAO rats was lower than that of SHAM rats at 21 days, which is within the above-mentioned time axis of CBF recovery.

However, the disadvantage of BCCAO model is that it only causes ischemia in the anterior cerebral circulation of the brain. Due to the compensation of the posterior circulation of the brain and the establishment of collateral circulation, it is usually difficult to achieve ideal cerebral ischemia by ligating the bilateral common carotid arteries, which affects the stability of the model. ${ }^{26}$ After the administration of SNP to reduce blood pressure, part of the blood supply of the posterior circulation is deprived, and the ischemia of the anterior circulation is further aggravated. ${ }^{27}$ The decrease in CBF caused by SNP may be the result of the reduction of systemic vascular resistance, resulting in a preferential blood flow to the low resistance systemic vascular bed rather than the cerebral vascular bed. ${ }^{28}$ Studies have shown that the BCCAO combined with SNP model shows obvious cognitive impairment in the MWM, step-down and eight-arm radial maze test. ${ }^{8,29}$ Clinically, SNP controlled blood pressure reduction does not affect the incidence of postoperative cognitive dysfunction in patients. ${ }^{30}$ In this study, the escape latency and swimming distance of BCCAO 

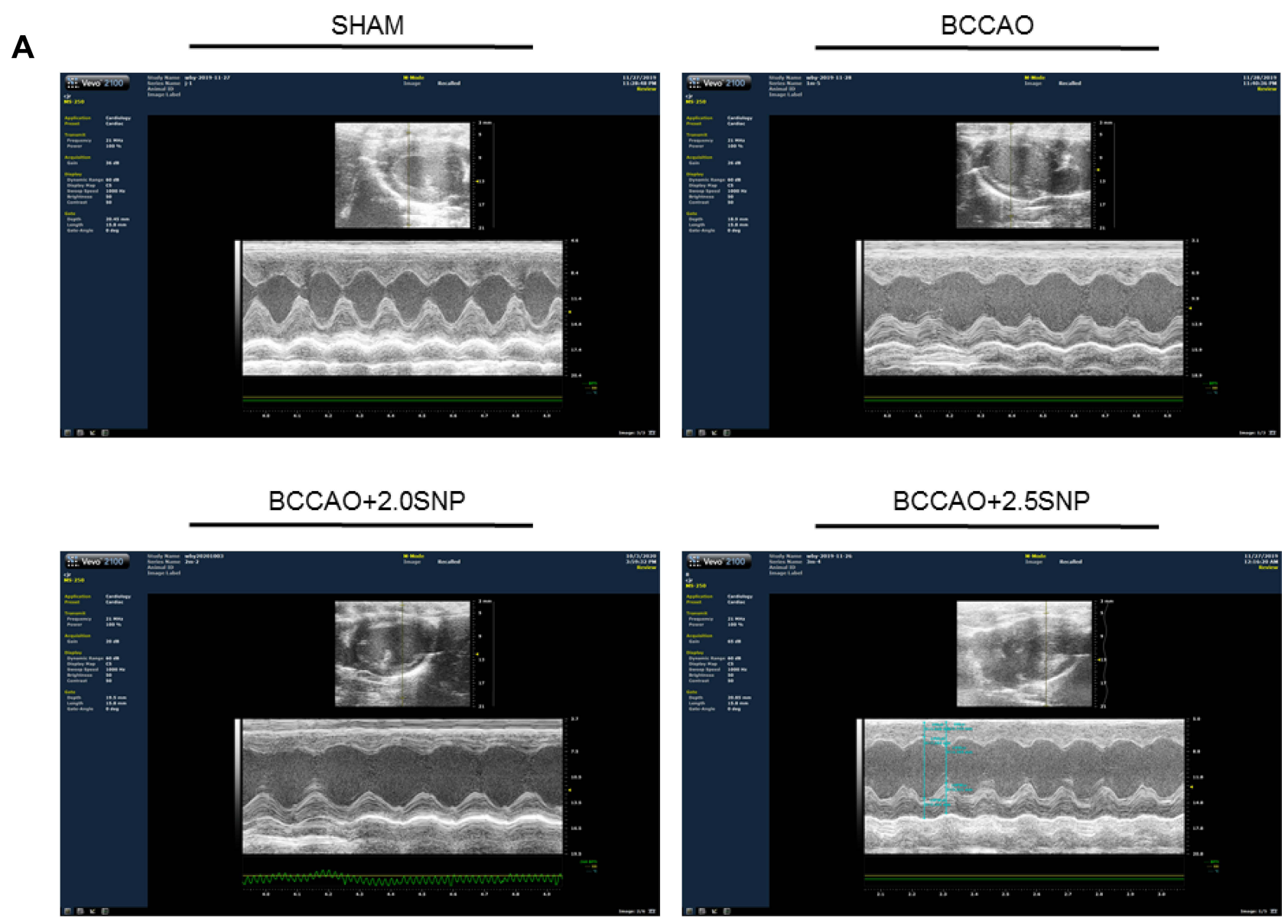

B

D

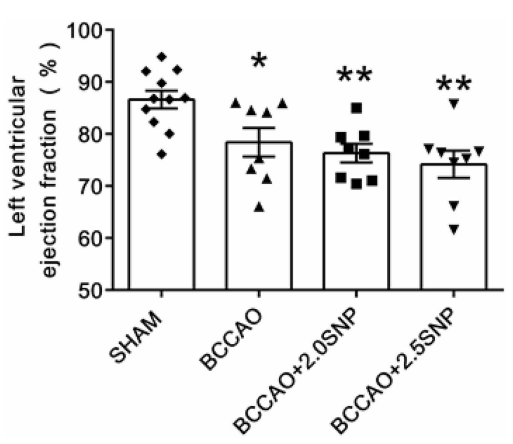

$E$
C

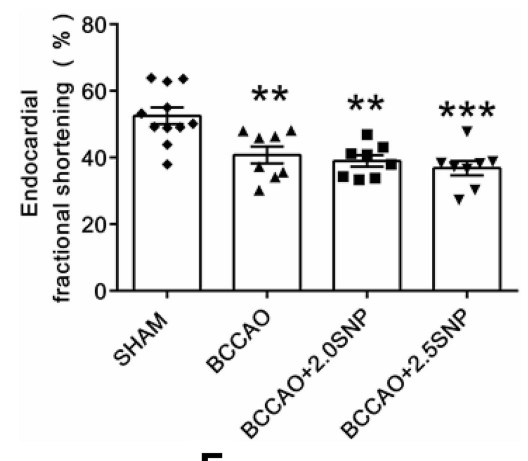

$\mathbf{F}$
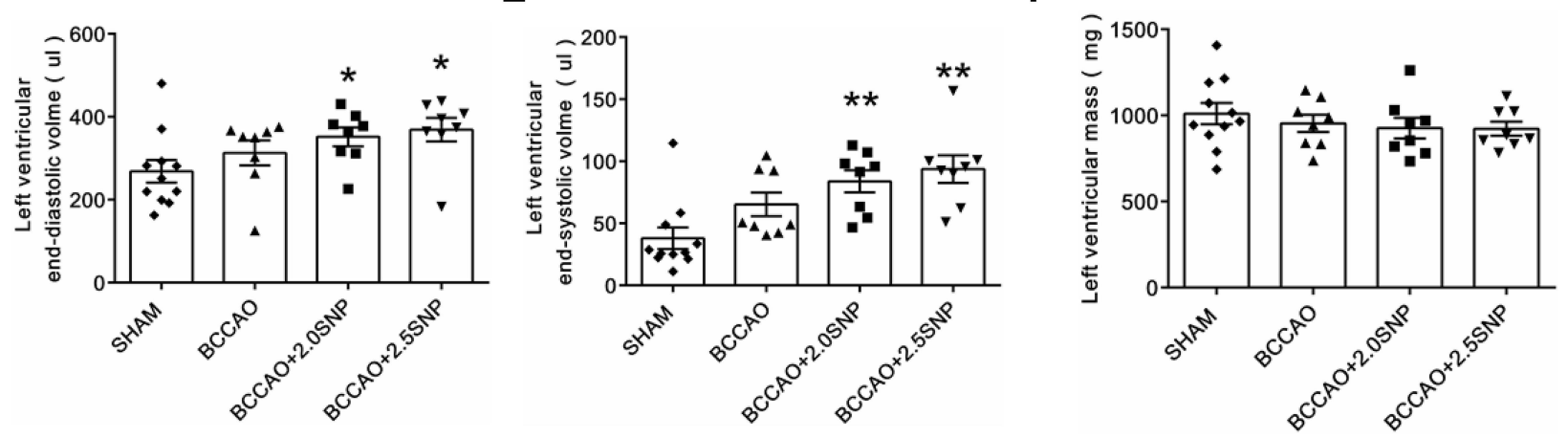

Figure 2 Evaluation of the left ventricular (LV) function by echocardiographic study. (A) Representative images of echocardiography in M-mode. (B) LV ejection fraction. (C) Endocardial fractional shortening. (D) LV end-diastolic volume. (E) LV end-systolic volume. (F) LV mass. The values are expressed as means \pm SEM. vs the SHAM group, $* \mathrm{P}<0.05,{ }^{*} \mathrm{P}<0.01,{ }^{*} * \mathrm{P}<0.001$. SHAM: $\mathrm{n}=1 \mathrm{I} ; \mathrm{BCCAO}: \mathrm{n}=8$; BCCAO+2.0SNP: $\mathrm{n}=8$; BCCAO+2.5SNP: $\mathrm{n}=8$. 
A
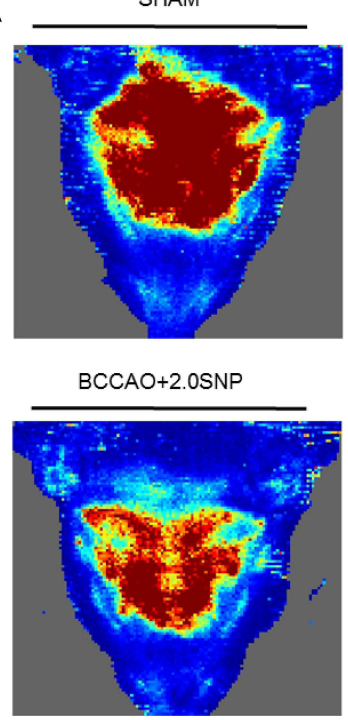

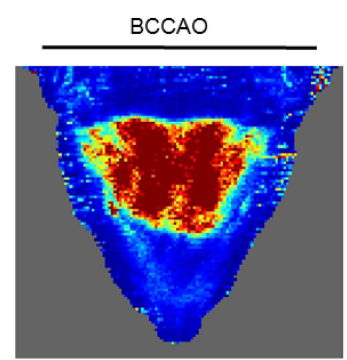

$\mathrm{BCCAO}+2.5 \mathrm{SNP}$

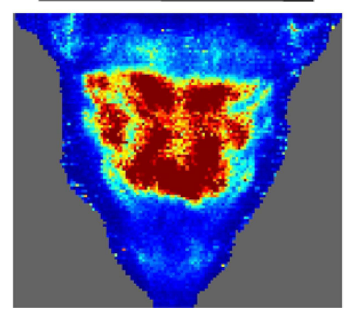

B

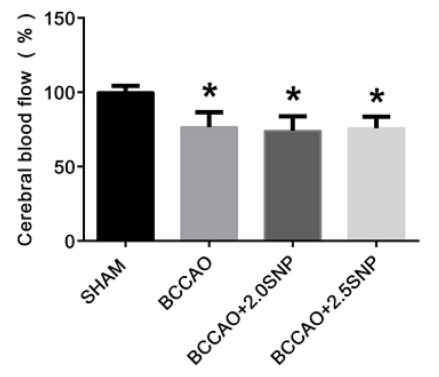

Figure 3 Cerebral blood flow (CBF) of rats measured by using laser speckle. (A) Representative CBF images. Red area: CBF; Blue area: non-CBF. (B) Quantitative analysis of CBF. The values are expressed as means \pm SEM. vs SHAM, *P<0.05. SHAM: $n=8 ; B C C A O: n=8 ; B C C A O+2.0 S N P: n=7 ; B C C A O+2.5 S N P: n=8$.

A

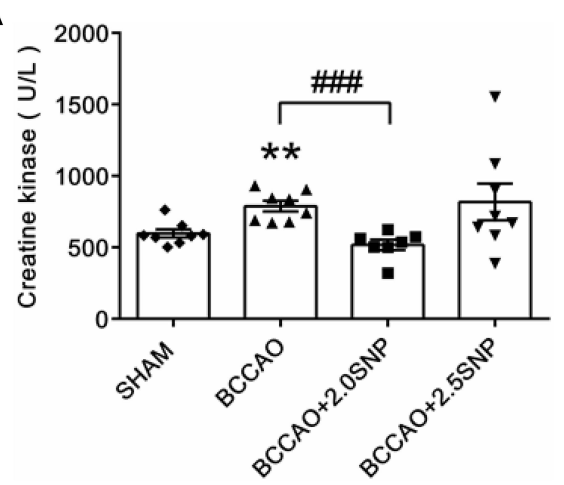

C

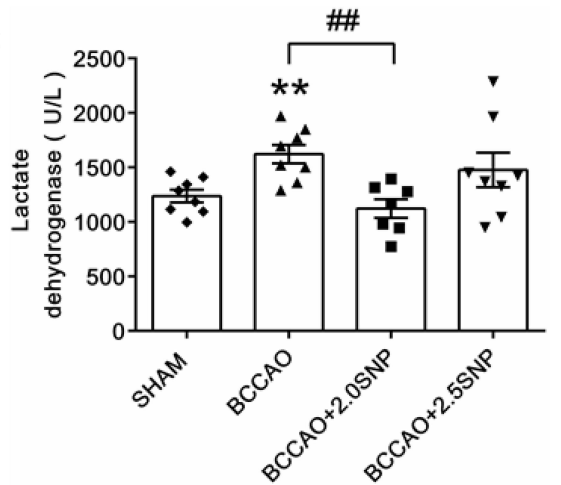

B

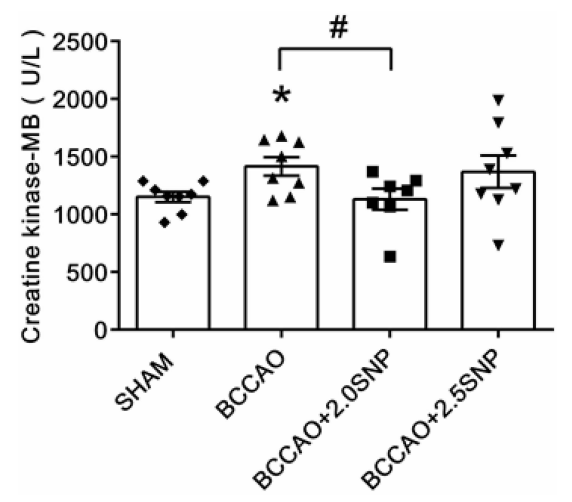

D

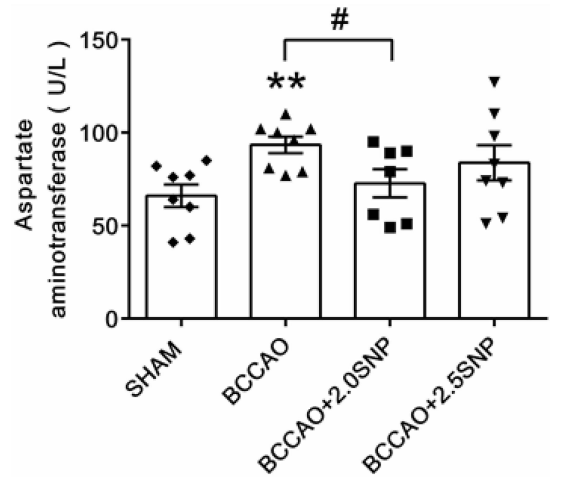

Figure 4 The levels of myocardial enzymes in serum. (A) Creatine kinase (B) Creatine kinase-MB. (C) Lactate dehydrogenase. (D) Aspartate aminotransferase. The values

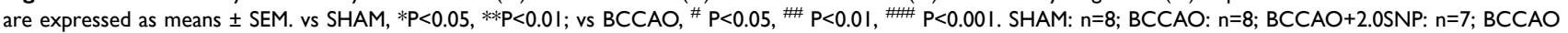
$+2.5 \mathrm{SNP}: \mathrm{n}=8$.

$+2.0 \mathrm{SNP}$ rats were longer than those of SHAM rats and BCCAO rats, and compared with SHAM rats, the time stayed in target quadrant and distance moved in target quadrant had a shortening trend, suggesting the spatial learning ability and reference memory of BCCAO +2.0SNP rats was impaired and the degree of spatial learning ability impaired was more serious than did of BCCAO rats. The escape latency and swimming distance 
of BCCAO+2.5SNP rats were longer than did of SHAM rats and BCCAO rats, the distance moved in target quadrant were shorter than did of SHAM rats, and the time stayed in target quadrant tended to be shorten, suggesting that the spatial learning ability and reference memory of $\mathrm{BCCAO}+2.5 \mathrm{SNP}$ rats was also impaired, and the BCCAO $+2.5 \mathrm{SNP}$ rats exhibited more stable cognitive dysfunction than $\mathrm{BCCAO}$ rats and $\mathrm{BCCAO}+2.0 \mathrm{SNP}$ rats. However, the swimming speed of BCCAO+2.5SNP rats was faster than that of SHAM rats, which may be the result of longer swimming distance and escape latency. Regrettably, no difference in cognitive impairment was observed between $\mathrm{BCCAO}+2.0 \mathrm{SNP}$ rats and $\mathrm{BCCAO}+2.5 \mathrm{SNP}$ rats. Moreover, despite being lower than SHAM rats, the CBF of $\mathrm{BCCAO}+2.0 \mathrm{SNP}$ rats and $\mathrm{BCCAO}+2.5 \mathrm{SNP}$ rats was not significantly different from that of $\mathrm{BCCAO}$ rats. It may be explained by the recovery of $\mathrm{CBF}$ due to the short halflife of SNP and the prolonged measurement timeline after surgery.

To the best of our knowledge, this is the first study to investigate impaired cardiac function in animal models of VD. The brain relies heavily on blood perfusion and consumes about $15 \%$ of cardiac output in a resting state. ${ }^{31}$ Hemodynamic balance in the heart-brain axis is increasingly recognized as a crucial factor in maintaining functional and structural integrity of the brain and thereby cognitive functioning. ${ }^{32}$ Once disturbed, it affects the blood supply of the brain and promotes the occurrence and development of cognitive impairment. For example, chronic heart failure leads to a $30 \%$ drop in $\mathrm{CBF}$ and is associated with common cognitive impairments involving attention and memory deficits. ${ }^{33}$ Meanwhile, neuro-cardiac feedback signals significantly promote aggravation and further progression of $\mathrm{HF}$ and are causal in the poor prognosis of heart failure. ${ }^{12}$ In this study, the EF and FS of rats in the three model groups were lower, suggesting that LV systolic dysfunction was insufficiency. The EDV and $\mathrm{ESV}$ of $\mathrm{BCCAO}+2.0 \mathrm{SNP}$ rats and $\mathrm{BCCAO}+2.5 \mathrm{SNP}$ rats enlarged, suggesting that $\mathrm{LV}$ expanded, and the systolic function may be worse than did of BCCAO rats. The loss of systolic function not only causes congestive manifestations of heart failure such as dyspnea and edema but also leads to poor cardiac remodeling, fibrosis, hypertrophy and aggravating the degree of cardiac dysfunction. ${ }^{34}$ However, there was no difference in LV mass of rats in the three model groups, which may suggest the LV systolic function in rats of VD was impaired, but not lost. As a vasoactive drug, short-term use of SNP has been clinically reported to relieve cardiac load and increase cardiac output. ${ }^{35}$ This study showed that the effect of a single intraperitoneal injection of SNP to temporarily increase cardiac output is not enough to affect the eventual decrease in cardiac output induced by the aggravation of CBF decline. In addition, oxidative damage of myocardium induces cell membrane to permeate or rupture, which results in leakage of cardiac enzymes into circulation. ${ }^{36}$ We found that the levels of myocardial enzyme in BCCAO rats were higher and had an increasing trend in BCCAO $+2.5 \mathrm{SNP}$ rats. Interestingly, the levels of myocardial enzymes in BCCAO+2.0SNP rats were lower than did in BCCAO rats, which of course requires further efforts to verify its reproducibility.

Our study has limitations. The detection of the levels of myocardial enzymes is of great significance to the assessment of myocardial damage. We found that the levels of myocardial enzymes in $\mathrm{BCCAO}+2.0 \mathrm{SNP}$ rats were lower than those of BCCAO rats and $\mathrm{BCCAO}+2.5 \mathrm{SNP}$ rats and were comparable to those of sham-operated rats. However, we assumed that this was an accidental deviation and did not further study and provide a reasonable explanation for this.

\section{Conclusions}

Our present study demonstrated that all of the three different VD rat models exhibited cognitive and cardiac function impairment. The BCCAO+2.0SNP model and BCCAO $+2.5 \mathrm{SNP}$ model damaged the spatial learning ability more seriously. The BCCAO+2.5SNP model caused more comprehensive cognitive impairment. In addition, the BCCAO +2.0SNP model and BCCAO+2.5SNP model might cause more serious damage to cardiac function. This study proves that the VD rat model causes cognitive dysfunction and at the same time damages the cardiac function, which provides a reference and basis for the treatment of VD via simultaneous treatment of heart and brain.

\section{Abbreviations}

VD, vascular dementia; BCCAO, permanent bilateral common carotid artery occlusion; CBF, cerebral blood flow; SNP, sodium nitroprusside; SHAM, sham-operated group; $\mathrm{BCCAO}+2.0 \mathrm{SNP}$, permanent bilateral common carotid artery occlusion combined with sodium nitroprusside $\left(2.0 \mathrm{mg} \cdot \mathrm{kg}^{-1}\right)$ group; BCCAO+2.5SNP, permanent bilateral common carotid artery occlusion combined with sodium nitroprusside $\left(2.5 \mathrm{mg} \cdot \mathrm{kg}^{-1}\right)$ group; MWM, Morris water maze; LV, left ventricular; DSWT, left ventricular diastolic septal wall thickness; SSWT, left ventricular systolic 
septal wall thickness; LVDD, left ventricular diastolic diameter; LVSD, left ventricular systolic diameter; DPWT, left ventricular diastolic posterior wall thickness; SPWT, left ventricular systolic posterior wall thickness; $\mathrm{EF}$, left ventricular ejection fraction; FS, left ventricular endocardial fractional shortening; EDV, left ventricular end-diastolic volume; ESV, left ventricular end-systolic volume; $\mathrm{CK}$, creatine kinase; CK-MB, creatine kinase$\mathrm{MB}$; LDH, lactate dehydrogenase; AST, aspartate aminotransferase.

\section{Ethics Approval Statement}

All animal procedures were conducted in accordance with protocols approved by the Ethics Committee for Animal Experimentation of Tianjin University of Traditional Chinese Medicine, Tianjin, China: TCM-LAEC20211125.

\section{Author Contributions}

All authors made a significant contribution to the work reported, whether that is in the conception, study design, execution, acquisition of data, analysis and interpretation, or in all these areas; took part in drafting, revising or critically reviewing the article; gave final approval of the version to be published; have agreed on the journal to which the article has been submitted; and agree to be accountable for all aspects of the work.

\section{Funding}

This work was supported by the financial support from the Natural Science Foundation of Tianjin (18JCQNJC83400) and the National Natural Science Foundation of China (No. 81503279).

\section{Disclosure}

The authors report no conflicts of interest in this work.

\section{References}

1. Wolters FJ, Ikram MA. Epidemiology of vascular dementia. Arterioscler Thromb Vasc Biol. 2019;39(8):1542-1549. doi:10.1161/ ATVBAHA.119.311908

2. Khan A, Kalaria RN, Corbett A, Ballard C. Update on vascular dementia. $J$ Geriatr Psychiatry Neurol. 2016;29(5):281-301. doi:10.1177/0891988716654987

3. Tuo QZ, Zou JJ, Lei P. Rodent models of vascular cognitive impairment. J Mol Neurosci. 2021;71(5):1-12. doi:10.1007/s12031020-01733-2

4. Xi Y, Wang M, Zhang W, et al. Neuronal damage, central cholinergic dysfunction and oxidative damage correlate with cognitive deficits in rats with chronic cerebral hypoperfusion. Neurobiol Learn Mem. 2014;109:7-19. doi:10.1016/j.nlm.2013.11.016
5. Peng XY, Wan T, Zhang LD. Overview about rat model studies of vascular dementia. Chin Arch Tradit Chin Med. 2018;36 (02):311-314.

6. Brown F, Hanlon K, Crockard H, et al. Effect of sodium nitroprusside on cerebral blood flow in conscious human beings. Surgical Neurology. 1977;7:67-70.

7. Henriksen L, Paulson OB. The effects of sodium nitroprusside on cerebral blood flow and cerebral venous blood gases. II. Observations in awake man during successive blood pressure reduction. European Journal of Clinical Investigation. 1982;12:389-393. doi:10.1111/ j.1365-2362.1982.tb00685.x

8. Zheng M, Qu L, Lou Y. Effects of icariin combined with Panax notoginseng saponins on ischemia reperfusion-induced cognitive impairments related with oxidative stress and CA1 of hippocampal neurons in rat. Phytother Res. 2008;22(5):597-604. doi:10.1002/ ptr.2276

9. Mariucci G, Stasi M, Taurelli R, et al. EEG power spectra changes and forebrain ischemia in rats. The Canadian Journal of Neurological Sciences. Le Journal Canadien Des Sciences Neurologiques. 2003;30 (1):54-60. doi:10.1017/s0317167100002444

10. Yang X, Kou S, Zheng K-S. [Effect of warmth-promotion needling on cerebral SOD, MDA and AChE in vascular dementia rats]. Acupuncture Res. 2007;32(3):170-173. Chinese.

11. Kim MS, Kim JJ. Heart and brain interconnection - clinical implications of changes in brain function during heart failure. Circ J. 2015;79(5):942-947. doi:10.1253/circj.CJ-15-0360

12. Doehner W, Ural D, Haeusler KG, et al. Heart and brain interaction in patients with heart failure: overview and proposal for a taxonomy. A position paper from the Study Group on Heart and Brain Interaction of the Heart Failure Association. Eur J Heart Fail. 2018;20(2):199-215. doi:10.1002/ejhf.1100

13. Liu DD, Yuan X, Chu SF, et al. CZ-7, a new derivative of Claulansine F, ameliorates 2VO-induced vascular dementia in rats through a Nrf2-mediated antioxidant responses. Acta Pharmacol Sin. 2019;40(4):425-440. doi:10.1038/s41401-018-0078-7

14. Wang J, Chen W, Wang Y. A ginkgo biloba extract promotes proliferation of endogenous neural stem cells in vascular dementia rats. Neural Regeneration Research. 2013;8(18):1655-1662. doi:10.3969/ j.issn.1673-5374.2013.18.003

15. Tian H, Ding N, Guo M, et al. Analysis of Learning and Memory Ability in an Alzheimer's Disease Mouse Model using the Morris Water Maze. J Vis Exp. 2019;152.

16. Redfors B, Ali A, Shao Y, et al. Different catecholamines induce different patterns of takotsubo-like cardiac dysfunction in an apparently afterload dependent manner. Int $J$ Cardiol. 2014;174 (2):330-336. doi:10.1016/j.ijcard.2014.04.103

17. Nagatani K, Nawashiro H, Takeuchi S, et al. Effects of a head-down tilt on cerebral blood flow in mice during bilateral common carotid artery occlusion. Asian Journal of Neurosurgery. 2012;7(4):171-173. doi:10.4103/1793-5482.106648

18. Duncombe J, Kitamura A, Hase Y, et al. Chronic cerebral hypoperfusion: a key mechanism leading to vascular cognitive impairment and dementia. Closing the translational gap between rodent models and human vascular cognitive impairment and dementia. Clin Sci (Lond). 2017;131(19):2451-2468. doi:10.1042/CS20160727

19. Du SQ, Wang XR, Xiao LY, et al. Molecular Mechanisms of Vascular Dementia: what Can Be Learned from Animal Models of Chronic Cerebral Hypoperfusion? Mol Neurobiol. 2017;54(5):3670-3682. doi:10.1007/s12035-016-9915-1

20. Zhang ZH, Shi GX, Li QQ, et al. Comparison of cognitive performance between two rat models of vascular dementia. Int J Neurosci. 2014;124(11):818-823. doi:10.3109/00207454.2014.880435

21. Jiwa NS, Garrard P, Hainsworth AH. Experimental models of vascular dementia and vascular cognitive impairment: a systematic review. J Neurochem. 2010;115(4):814-828. doi:10.1111/j.14714159.2010.06958.x 
22. Bhatia P, Singh N. Ameliorative Effect of Phosphodiesterase-5 Inhibitor in Rat Model of Vascular Dementia. Current Neurovascular Research. 2019;16(1):27-39. doi:10.2174/ 1567202616666190130153954

23. Bhatia P, Kaur G, Singh N. Ozagrel a thromboxane A2 synthase inhibitor extenuates endothelial dysfunction, oxidative stress and neuroinflammation in rat model of bilateral common carotid artery occlusion induced vascular dementia. Vascular Pharmacology. 2021;137:106827. doi:10.1016/j.vph.2020.106827

24. Neto CJBF, Paganelli RA, Benetoli A, Lima KCM, Milani H. Permanent, 3-stage, 4-vessel occlusion as a model of chronic and progressive brain hypoperfusion in rats: a neurohistological and behavioral analysis. Behav Brain Res. 2005;160(2):312-322. doi:10.1016/j.bbr.2004.12.016

25. Gooch J, Wilcock DM. Animal Models of Vascular Cognitive Impairment and Dementia (VCID). Cell Mol Neurobiol. 2016;36 (2):233-239. doi:10.1007/s10571-015-0286-3

26. Hu R, Zhong X. The Summary of Researching on the Animal Model of Mice with Vascular Dementia. $J$ Zhejiang Univ Med Sci. 2008;1:137-138+140.

27. Ma DY, Fang JP, Zhu LJ, Han ZY. Comparison of three rat models of synthetic vascular dementia. $J$ Beijing Univ Tradit Chin Med. 2011;34(12):832-35+74.

28. Immink RV, van den Born BJ, van Montfrans GA, et al. Cerebral hemodynamics during treatment with sodium nitroprusside versus labetalol in malignant hypertension. Hypertension. 2008;52 (2):236-240. doi:10.1161/HYPERTENSIONAHA.108.110395

29. Wang J, Chen W, Wang Y-L. [The long-term dynamic changes of proliferation and differentiation of neural stem cells in subventricular zone and dentate gyrus of vascular dementia rats]. Chin J App Physiol. 2013;29(2):170-173. Chinese.
30. Liu P, Diao S, Sun H, et al. Effects of sodium nitroprusside controlled blood pressure reduction on cognitive function and cerebral oxygen metabolism in elderly hypertensive patients after hip replacement. J Clin Anesth. 2009;25:500-501.

31. Smith KJ, Ainslie PN. Regulation of cerebral blood flow and metabolism during exercise. Exp Physiol. 2017;102(11):1356-1371. doi:10.1113/EP086249

32. Hooghiemstra AM, Bertens AS, Leeuwis AE, et al. The Missing Link in the Pathophysiology of Vascular Cognitive Impairment: design of the Heart-Brain Study. Cerebrovasc Dis Extra. 2017;7(3):140-152. doi:10.1159/000480738

33. Ackerman RJS. Cerebral blood flow and neurological change in chronic heart failure. Stroke. 2001;32(11):2462-2464.

34. Connelly KA, Zhang Y, Desjardins JF, et al. Load-independent effects of empagliflozin contribute to improved cardiac function in experimental heart failure with reduced ejection fraction. Cardiovasc Diabetol. 2020;19(1):13. doi:10.1186/s12933-020-0994-y

35. Guo B, Yang T, Nan J, et al. Efficacy and safety of Shenfu injection combined with sodium nitroprusside in the treatment of chronic heart failure in patients with coronary heart disease: a protocol of randomized controlled trial. Medicine. 2021;100:e24414. doi:10.1097/ MD.0000000000024414

36. Akila P, Vennila L. Chlorogenic acid a dietary polyphenol attenuates isoproterenol induced myocardial oxidative stress in rat myocardium: an in vivo study. Biomed Pharmacother. 2016;84:208-214. doi:10.1016/j.biopha.2016.09.028
Neuropsychiatric Disease and Treatment

\section{Publish your work in this journal}

Neuropsychiatric Disease and Treatment is an international, peerreviewed journal of clinical therapeutics and pharmacology focusing on concise rapid reporting of clinical or pre-clinical studies on a range of neuropsychiatric and neurological disorders. This journal is indexed on PubMed Central, the 'PsycINFO' database and CAS, and

\section{Dovepress}

is the official journal of The International Neuropsychiatric Association (INA). The manuscript management system is completely online and includes a very quick and fair peer-review system, which is all easy to use. Visit http://www.dovepress.com/testimonials.php to read real quotes from published authors. 